\title{
Evaluación del aprendizaje combinado para la enseñanza de Cirugía Bucal en alumnos de pregrado de Odontología
}

\section{Berta García ${ }^{1}$ y Verónica Odeh ${ }^{2}$}

${ }^{1}$ Profesor asocido de Cirugía Oral. Master en Cirugía Oral e Implantología, Facultad de Medicina y Odontología de la Universidad de Valencia. Valencia, España.

${ }^{2}$ Estudiante de pregrado de Odontología, Facultad de Medicina y Odontotología de la Universidad de Valencia. Valencia, España.

\begin{abstract}
Dentistry students not only have to gain theorical knowledge, but also practical skills. For these reasons it becomes increasingly important to investigate the potential of alternative learning tools. The aim of the study was to compare the efficacy of blended learning versus conventional learning for the teaching of Oral Surgery in terms of academic results, quality, satisfaction and motivation of students. To do this, the sample was divided into a GE and $G C$ that jointly attended a theorical class and, subsequently, completed the training with the visualization of videos in a web platform and with the reference book, respectively. The GE scored better on the practical exam ( $p$ $<0.001)$, had more positive opinions about training, felt more satisfied and more motivated than the $C G$.
\end{abstract}

Keywords: Oral surgery, anesthesia, new technologies, combined learning.

\begin{abstract}
Resumen
Los estudiantes de Odontología no sólo tienen que adquirir conocimientos teóricos, sino también habilidades prácticas. Por estas razones se hace cada vez más importante investigar el potencial de las herramientas de aprendizaje alternativas. El objetivo del estudio fue comparar la eficacia del aprendizaje combinado frente al convencional para la enseñanza de la Cirugía Bucal, en términos de resultados académicos, calidad, satisfacción y motivación de los alumnos. Para ello, se divide la muestra en un GE y GC que asistieron conjuntamente a una clase teórica y, posteriormente, completaron la formación con la visualización de vídeos en una plataforma web y con el libro de referencia, respectivamente. El GE obtuvo mejores calificaciones en el examen práctico $(p<0,001)$, tuvieron opiniones más positivas sobre la formación, se sintieron más satisfechos y mas motivados que el GC.
\end{abstract}

Palabras clave: cirugía bucal, anestesia, nuevas tecnologías, aprendizaje combinado. 


\section{Introducción}

La enseñanza en el campo de la salud presenta un gran reto para los alumnos, quienes tienen que adquirir no solo conocimientos teóricos, sino también las habilidades prácticas necesarias para su ejercicio profesional. Estas habilidades requieren de una enseñanza preclínica y clínica, con la atención a pacientes reales. Pero uno de los problemas que surge con mayor frecuencia en la enseñanza de la Medicina y la Odontología, es la dificultad de enfrentar al alumno al número de pacientes que asegure su formación idónea. Al no ser siempre posible la formación completa con pacientes, se complementa con otros recursos que limitan la comprensión de los conceptos más difíciles y el traslado de la teoría a la práctica. Así, la enseñanza clínica resulta, en ocasiones, complicada y dificulta la oportunidad de desarrollar competencias clínicas.

Los avances tecnológicos de los últimos años y el desarrollo acelerado de las Tecnologías de la Información y la Comunicación (TIC), han dado solución a estos problemas. La introducción del aprendizaje asistido por ordenador, con resolución de casos, ejercicios interactivos, videos demostrativos, simuladores y plataformas de Internet (Web, foros, etc.) constituyen un recurso docente muy útil para optimizar la enseñanza práctica. Permiten crear entornos de aprendizaje más cercanos a la realidad, fomentar la interacción y el aprendizaje práctico, presentando la información de una manera más atractiva y manteniendo al alumno motivado durante su proceso de formación. Tanto es así que, aunque el uso de las tecnologías informáticas no reemplaza los escenarios clínicos reales, constituyen una herramienta adicional y complementaria que facilita la adquisición de conocimientos y habilidades prácticas.

La utilización de estos nuevos medios debe entenderse siempre como apoyo y complemento a los modelos de enseñanza clásicos, que han demostrado durante años ser eficientes para la formación del alumnado. La combinación de los métodos de enseñanza tradicionales con las modernas tecnologías de la información, se conoce como aprendizaje combinado.

En el área de la Odontología, se están empleando cada vez más las nuevas tecnologías de la información, pero son pocos los trabajos que se han dedicado a diseñar y promover una propuesta que innove los tradicionales modelos de enseñanza. Se han encontrado trabajos publicados sobre innovación docente en disciplinas de la Odontología como son Radiología para el diagnóstico (Meckfessel y cols, 2011; Kavadella y cols, 2011; Santos y cols, 2016) y Ortodoncia (Aly y cols, 2011; Linjawi y cols, 2009), en los que se observa una mejora en los resultados académicos y respuestas positivas en cuanto a eficacia, motivación y compromiso activo. Sin embargo, la bibliografía publicada con respecto a la Cirugía Oral en particular es poca o casi nula. Solo se encontró un estudio en el que se evaluó un programa de simulación basado en una Web para enseñar razonamiento clínico y evaluación de pacientes en Cirugía Oral de pregrado (Kruger, 2016). El estudio concluyó que era una herramienta valiosa, mejorando los resultados de aprendizaje, en comparación con la enseñanza tradicional por sí sola. Es por ello que se lleva a cabo el presente estudio, donde se trató de evaluar cuán efectivo resulta el uso de una página web con vídeos interactivos, como apoyo y complemento para la enseñanza de la Cirugía Oral. 


\section{Objetivos}

En un grupo de estudiantes de pregrado de Odontología de la Universitat de València, que fueron sometidos al aprendizaje combinado frente al convencional, para la enseñanza de Cirugía Bucal, se plantearon los siguientes objetivos:

1. Estudiar los resultados académicos.

2. Evaluar la motivación de los estudiantes.

3. Conocer las opiniones y actitudes frente a la metodología empleada.

\section{Desarrollo de la innovación}

\subsection{Diseño del estudio}

Se realizó, en el Departamento de Cirugía Bucal de la Universitat de València, entre septiembre de 2016 y Mayo de 2017, un estudio para valorar el impacto del aprendizaje combinado mediante material multimedia (web: www.cirubuca.wixsite.com/ciruvestic) en alumnos de pregrado de Odontología. El estudio fue aprobado por el comité de ética de la Universitat de València H1486051731594. A todos los participantes se les explicó previamente en qué consistía el estudio y se les pidió firmar un consentimiento informado para participar en el mismo.

Se estableció un grupo de estudio (GE) o grupo de aprendizaje combinado, en el que se introdujo material didáctico en línea y un grupo de aprendizaje convencional, que sirvió de grupo control (GC). Los participantes fueron asignados de manera aleatoria.

\subsection{Población de estudio}

Los cristerios de inclusión fueron:

- Alumnos de pregrado de Odontología de la Universidad de Valencia.

- Disponibilidad horaria

- Disponer de un ordenador y auriculares por persona para poder visualizar los vídeos, en el caso del grupo de aprendizaje combinado.

- Firma de un consentimiento informado tras recibir información acerca del estudio. Se excluyeron de la muestra aquellos alumnos que no hubiesen completado los cuestionarios en su totalidad.

De un total de 81 alumnos que se inscribieron inicialmente a la clase, solo se presentaron 55 alumnos, de los cuales 2 fueron excluidos por no haber rellenado por completo los cuestionarios. Finalmente, un total de 53 alumnos cumplieron todos los criterios de inclusión y fueron incluidos en este estudio, de los cuales 25 asistieron a la enseñanza convencional y 28 a la combinada. 


\subsection{Material}

\section{Material didáctico:}

1. Una presentación de Keynote de 37 diapositivas sobre anatomía, anestesia local y exodoncia simple de dientes mandibulares, conteniendo dibujos esquemáticos, imágenes clínicas y cuadros-resumen, así como textos cortos, simples y fáciles de leer.

2. El capítulo 6 "Técnicas anestésicas primarias" del libro "Anestesia local en odontología” escrito por Miguel Peñarrocha, Jose Ma Sanchís y Jose Ma Martínez ${ }^{29}$, junto con imágenes en 2D.

3. Práctica 5 "Introducción a la exodoncia” de la libreta de prácticas de Cirugía Bucal de $3^{\circ}$ de Odontología de la Universidad de Valencia.

4. Una página Web (Fig.1-2) de elaboración propia, que fue presentada en el curso académico 2014-2015 como proyecto ESTIC, titulado "Educación asistida por ordenador: aplicación de las nuevas tecnologías en el proceso de aprendizaje de la anestesia dental y la cirugía bucal", con el código ESTIC1415_09 y bajo la convocatoria recogida en la Resolución del Vicerrector de Políticas de Formación y Calidad Educativa, del 8 de Octubre de 2014, obteniendo el reconocimiento de grupo ESTIC de excelencia. La Web incluye herramientas como imágenes, vídeos, cuestionarios de autoevaluación, plataforma de discusión en línea para exposición y resolución de dudas y acceso a enlaces externos.

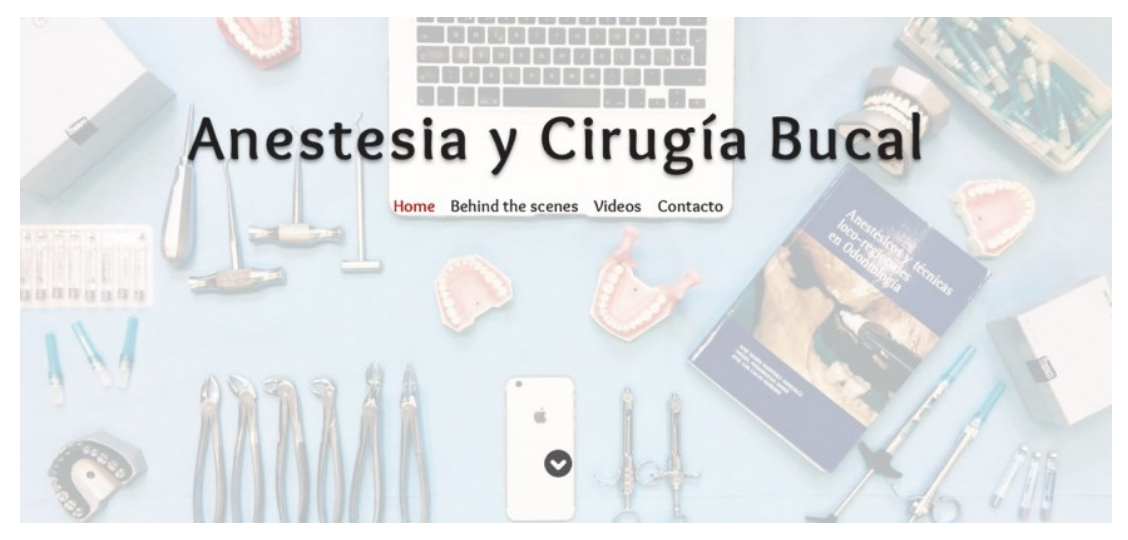

Fig.1 Página principal de la web.

5. Fantomas e instrumental de anestesia y extracción (jeringas, botadores y fórceps) cedidos por el Departamento de Cirugía Bucal de la Facultad de Medicina y Odontología de la Universitat de València.

Material para la evaluación y recogida de datos: Cuestionarios, fantomas de anestesia y extracción e instrumental quirúrgico (jeringas de anestesia, botadores, fórceps, etc.) 


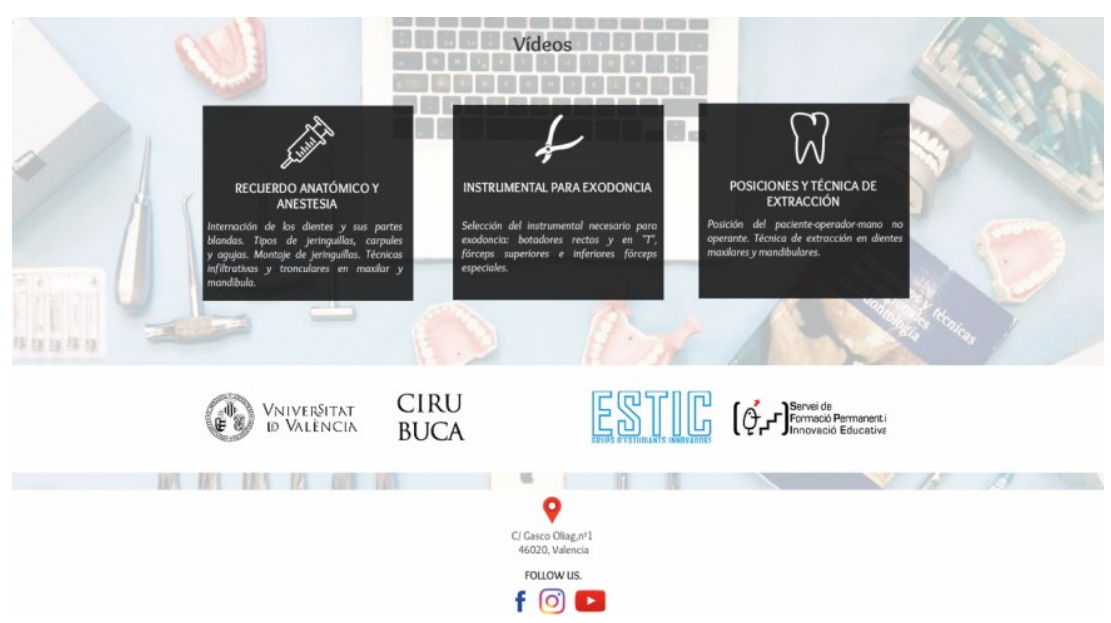

Fig.2 Menú principal de los videos, con acceso directo a cada sección pichando sobre el icono.

\subsection{Procedimiento}

Para evaluar la eficacia del aprendizaje combinado frente al convencional, se siguió el procedimiento empleado por otros autores (Hu J y cols, 2008) en un estudio similar. A continuación, se esquematiza el procedimiento llevado a cabo para el aprendizaje y la recogida de datos (Fig.3).

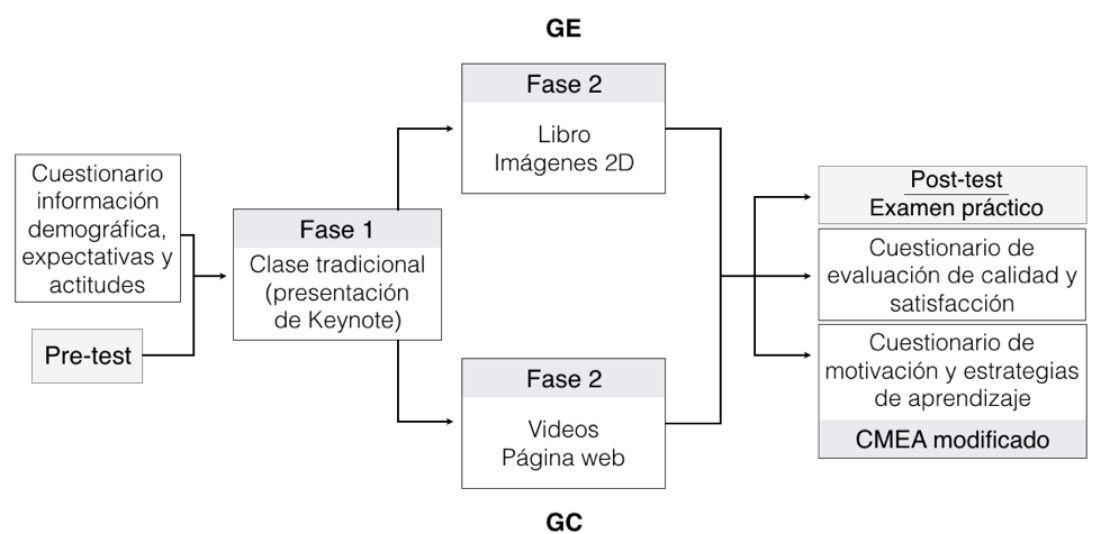

Fig. 3 Procedimiento de la investigación

\subsubsection{Actividad formativa}

La actividad de formación consistió en un curso de una hora de duración, que fue desarrollado especialmente para este propósito, que no modificó el plan de estudios y que se llevó a cabo dentro del horario de los estudiantes. El curso se dividió en dos tiempos. En primer lugar, ambos grupos asistieron conjuntamente a una clase tradicional, con el apoyo de una presentación de Keynote, que duró aproximadamente 30 minutos. 
Fue impartida por el profesor encargado de la asignatura (BGM), que acostumbra a dar la clase sobre el tema específico del que se trató.

Una vez finalizada la clase teórica, se aleatorizó y dividió a todos los pacientes en GE y GC. De tal manera que, en una segunda fase, de otros 30 minutos de duración, se entregó material complementario para el aprendizaje:

- En el grupo control (GC), el material complementario se compuso del capítulo 6 "Técnicas anestésicas primarias" del libro "Anestesia local en odontología" y la práctica 5 "Introducción a la exodoncia" de la libreta de prácticas de Cirugía Bucal de $3^{\circ}$ de Odontología de la Universidad de Valencia.

- En el grupo estudio (GE), los alumnos navegaron por la página web "Anestesia y cirugía bucal" (www.cirubuca.wixsite.com/ciruvestic), dirigidos por VOO para evitar el riesgo de un conocimiento incompleto.

\subsubsection{Protocolo de evaluación y recogida de datos}

Todos los estudiantes completaron cuestionarios, de la siguiente manera: (A) antes de iniciar el curso, ambos grupos completaron (i) un cuestionario de información demográfica, expectativas y actitudes y (ii) una prueba de evaluación de conocimientos teóricos (pre-test); (B) al final del curso, ambos grupos completaron (i) una prueba de conocimiento (post-test), (ii) un examen práctico sobre fantomas de anestesia y extracción, (iii) un cuestionario de calidad de la actividad formativa y satisfacción y (iv) un cuestionario de motivación y estrategias de aprendizaje.

Los cuestionarios fueron establecidos en base a los encontrados en estudios educativos similares (Kavadella y cols, 2011; Santos y cols, 2016; Hu J y cols, 2008; Varthis y cols, 2016; Linjawi y cols; 2009; Neuhaus y cols, 2008; Zambrano y cols, 2016). Fueron los mismos para ambos grupos, a excepción del cuestionario de calidad, en el que los estudiantes del grupo de aprendizaje combinado respondieron a una serie de preguntas adicionales.

\subsection{Análisis estadístico}

Todos los datos fueron almacenados en un documento Excel y procesados estadísticamente. Los análisis se realizaron con el programa SPSS 15.0 para Windows. Para la comparación del GE y GC se utilizó la prueba de Kolmogorov-Smirnov que evaluó las puntuaciones en los test de conocimiento y el modelo no paramétrico de Brunner-Langer para comparar la puntuación pre y post-test del examen teórico de ambos grupos. Se evaluó si mejoró el conocimiento de uno y otro grupo en la misma magnitud mediante un estadístico tipo ANOVA (ATS). La comparación de las puntuaciones en escala Likert se realizó mediante la prueba de Mann-Whitney (MW), por ejemplo, para contrastar el grado de acuerdo con los ítems de calidad de la actividad formativa y satisfacción o con los del CMEA. Para comprobar si las respuestas dependían de haber formado parte del GE o GC, se empleó el test $\mathrm{Chi}^{2}$ de asociación. El nivel de significancia empleado en los análisis fue del $5 \%(\alpha=0.05)$.

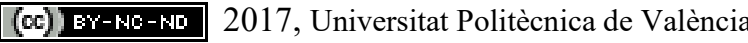


Se desarrolló un estudio previo del tamaño muestral, seleccionando como variable primaria la nota del examen de conocimientos teóricos al finalizar la formación (post-test). Para determinar la diferencia entre las notas medias del grupo estudio y grupo control que quisimos detectar como estadísticamente significativo, se consultó la literatura ${ }^{3}$, encontrándose notas medias de $6,9 \pm 1,4$ y $8,1 \pm 1,4$ en dos grupos similares control y estudio. Ello supone una diferencia media de 1,2 puntos y, por tanto, un tamaño de efecto grande. Mediante un t-test (Tabla 1), se concluyó que eran necesarios 52 alumnos para alcanzar una potencia del $80 \%$ con el objeto de detectar una diferencia en la nota media de 1,2 puntos y una desviación estándar de 1,4 puntos. Se asumió una confianza del 95\% para un t-test de grupos independientes.

Tabla 1. Tamaño muestral total necesario según diferencia mínima relevante para la nota media y potencia (t-test). Calculado para un nivel de confianza del $95 \%$.

\begin{tabular}{lllll}
\hline Medias para la diferencia & Tamaño efecto & \multicolumn{3}{l}{ Potencia alcanzada } \\
\cline { 3 - 5 } mínima relevante & (d) & $\mathbf{6 0 \%}$ & $\mathbf{7 0 \%}$ & $\mathbf{8 0 \%}$ \\
$\mathbf{6 , 9}-\mathbf{7 , 2}$ & $\mathbf{0 , 2}$ (pequeño) & 492 & 620 & 788 \\
$\mathbf{6 , 9}-\mathbf{7 , 6}$ & $\mathbf{0 , 5}$ (medio) & 82 & 102 & 128 \\
$\mathbf{6 , 9}-\mathbf{8 , 1}$ & $\mathbf{0 , 8}$ (grande) & 34 & 42 & $\mathbf{5 2}$ \\
\hline
\end{tabular}

\section{Resultados}

\subsection{Resultados académicos}

\subsubsection{Pre-test/post-test}

Antes de la formación, los alumnos del GC consiguieron una nota media 4,1 $\pm 1,3$ frente a $3,9 \pm 1,5$ en el GE. Se trata de una mínima diferencia media de 0,2 puntos. Tras la formación, las notas medias se elevaron a $6,1 \pm 1,4$ y $6,6 \pm 1,2$ en GC y GE respectivamente. La diferencia fue, entonces, de 0,5 puntos a favor del GE (Fig.4).

Para evaluar si el incremento de nota en uno y otro grupo fue comparable, se estimó un modelo de Brunner-Langer (Tabla 2), donde se observó la mejora del nivel de conocimientos que supuso participar en la acción formativa $(p<0,001)$, siendo mayor en el grupo estudio $(\mathrm{p}=0,054)$, que en el grupo control.

\subsubsection{Examen práctico}

La nota media de la parte práctica se elevó a 8,1 $\pm 1,1$ en el GE y a 6,9 $\pm 1,2$ del grupo GC (Fig.5), siendo las diferencias estadísticamente significativas $(\mathrm{p}<0,001)$ para la prueba MW. 


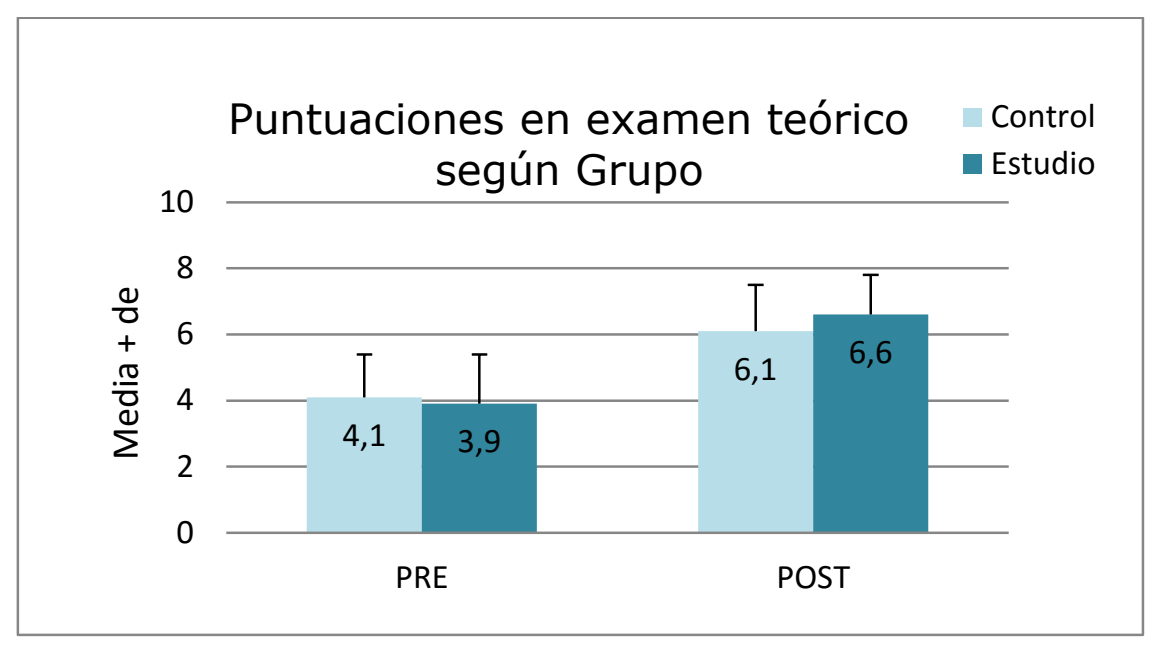

Fig.4 Puntuaciones del examen teórico según grupo

Tabla 2. Cambios en la distribución de notas teóricas de los grupos: resultados modelo de Brunner-Langer y test ATS tipo ANOVA.

\begin{tabular}{|c|c|}
\hline & p-valor \\
\hline Tiempo & $<0,001 * * *$ \\
\hline Grupo & 0,549 \\
\hline Interacción & 0,054 \\
\hline
\end{tabular}

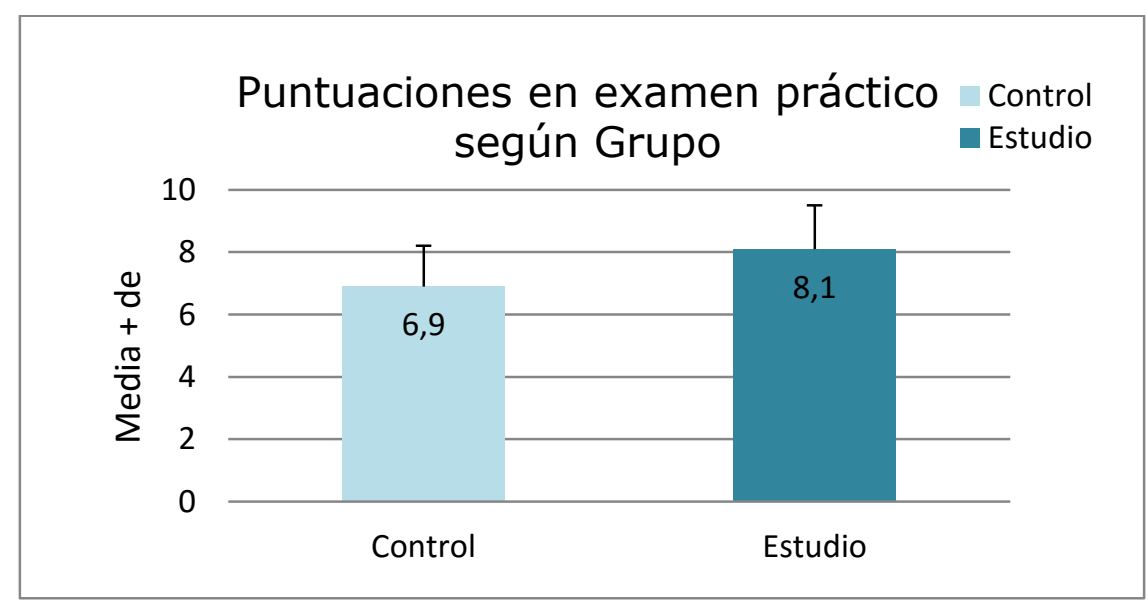

Fig.5 Puntuaciones en examen práctico según grupo

(cc) EY-NC-ND 2017, Universitat Politècnica de València 
La tabla 3 describe la evaluación a cada uno de los 5 ítem que componen la parte de anestesia y los 5 de la parte de extracción, donde se observa en qué aspectos en concreto se maximizaron las diferencias entre grupos. El 53,6\% en el GE realizó la aspiración, frente a sólo el 32\% en el GC ( $\mathrm{p}<0,025)$. El 71,4\% de los participantes del GE cogieron correctamente el botador, frente a sólo el $36 \%$ de los controles $(p<0,001)$. Asimismo, el 75\% del GE colocó correctamente la mano no operante frente a sólo el $40 \%$ de los controles $(p<0,001)$. Una batería de test de Fisher identifica las diferencias más significantes.

Tabla 3. Proporción de actuaciones correctas según grupo: resultados test exacto de Fisher (Fis).

\begin{tabular}{rcc}
\hline & p-valor \\
\hline ANESTESIA & Montaje jeringa & 0,418 \\
Forma coger jeringa & 0,633 \\
Entrada desde premolares & 1,000 \\
Lugar inyección & 0,091 \\
Realiza aspiración & $\mathbf{0 , 0 2 5 *}$ \\
EXTRACCIÓN & Selección instrumental & 0,145 \\
& Forma coger botador & $<\mathbf{0 , 0 0 1 * * *}$ \\
Forma coger fórceps & 0,418 \\
& Posición mano no operante & $<\mathbf{0 , 0 0 1 * * *}$ \\
& Técnica de extracción & 0,905 \\
\hline
\end{tabular}

${ }^{*} \mathrm{p}<0,05 ; \quad * * \mathrm{p}<0,01 ; \quad * * * \mathrm{p}<0,001$

\subsection{Evaluación de calidad de la actividad formativa y satisfacción}

\subsubsection{Calidad de la actividad formativa}

Con respecto a la opinión general de la clase, se encontró un porcentaje de satisfacción muy similar en ambos grupos: $84 \%$ el GC y $89,3 \%$ el GE, correspondiente a las valoraciones buenas y muy buenas (códigos 4 y 5 de la escala de Likert). Sin embargo, si atendemos sólo al máximo grado de satisfacción (código 5 de la escala de Likert), el 50\% del GE asignó esta categoría, frente a solo el $20 \%$ del GC. Estas diferencias fueron estadísticamente significativas $(\mathrm{p}=0,003, \mathrm{MW})$.

Con respecto a cuestiones específicas relacionadas con la estructura y materiales de la docencia recibida, aspectos como la organización, el diseño y la cobertura integral del tema fueron valorados positivamente por, al menos, el 80\% del GC. En el GE, la cifra se elevó al $92 \%$, destacando los aspectos de contenido, organización, material y facilidad para entender. 
Además, el 32\% del GC se mostró indiferente (código 3 de la escala Likert) cuando se preguntó sobre la facilidad para entender los contenidos, frente a solo el 7,1\% del GE.

El GE, además, valoró las hipotéticas ventajas del aprendizaje combinado frente a los métodos de enseñanza convencionales que conocían. La mayoría de ellos estuvo de acuerdo o muy de acuerdo (códigos 4 y 5 de la escala Likert) en que el aprendizaje combinado es más efectivo y motivador, promueve un compromiso activo para el aprendizaje individual y la auto-evaluación y mejora el pensamiento crítico.

\subsubsection{Satisfacción}

El 76\% del GC consideró que la clase fue una experiencia formativa agradable e instructiva (36\% muy de acuerdo $+40 \%$ de acuerdo), frente $96,4 \%$ del GE (50\% muy de acuerdo + $46,4 \%$ de acuerdo). El $72 \%$ del GC recomendaría la experiencia a compañeros ( $24 \%$ muy de acuerdo $+48 \%$ de acuerdo), frente al 100\% del GE ( $75 \%$ muy de acuerdo $+25 \%$ de acuerdo), que también tuvo intención de seguir utilizando la web para su aprendizaje.

\subsection{Motivación y estrategias de aprendizaje}

Los resultados del cuestionario de motivación y estrategias de aprendizaje (CMEA) se presentan haciendo referencia, en primer lugar, a la escala de motivación y, posteriormente al uso de estrategias de aprendizaje.

\subsubsection{Escala de motivación}

El análisis de la motivación de los estudiantes se realizó en base a las respuestas de los 18 ítems de la escala de motivación del CMEA. Como se mencionó, estos ítems están agrupados en 5 sub-escalas que evalúan aspectos motivacionales diferentes. Los resultados presentan, en términos de medias (rango de variación entre 1 y 5 ), el grado de acuerdo con los diferentes ítems. El GE presentó mayor motivación que el GC ( $\mathrm{p}<0,034)$, especialmente en las secciones de valoración de la tarea $(\mathrm{p}<0,001)$ y control de autoeficacia $(\mathrm{p}<0,013)$ (Tabla 4$)$.

Tabla 4. Puntuaciones sub-escala y escala de motivación del CMEA según grupo: resultados test Mann-Whitney (MW).

\begin{tabular}{rc}
\hline & p-valor \\
\hline ORIENTACIÓN META INTRÍNSECA & 0,770 \\
ORIENTACIÓN META EXTRÍNSECA & 0,872 \\
VALOR DE LA TAREA & $\mathbf{0 , 0 0 1 * *}$ \\
CONFIANZA EN CONTROL DEL APRENDIZAJE & 0,971 \\
CONTROL DE AUTOEFICACIA & $\mathbf{0 , 0 1 3 *}$ \\
APRECIACIÓN GENERAL & $\mathbf{0 , 0 3 *}$ \\
\hline $\mathrm{p}<0,05 ; \quad * * \mathrm{p}<0,01 ; \quad * * * \mathrm{p}<0,001$ &
\end{tabular}

(cc) EY-NC-ND 2017, Universitat Politècnica de València 
Los resultados fueron similares en ambos grupos con respecto a la orientación de meta intrínseca y meta extrínseca. Sin embargo, el GE evidenció una alta valoración de las tareas, donde la media adoptó un valor de 4,54 frente a 4,1 en el GC $(\mathrm{p}<0,001)$. Los alumnos del GE se sintieron, tras la clase, más capaces y con mayor confianza para afrontar sus prácticas clínicas, con una media de 4,46 frente a 3,68 del GC ( $\mathrm{p}<0,001)$ y creyeron que el material de la clase fue útil para su aprendizaje, con una media de 4,71 en comparación con una media de 4,16 en el GC $(p<0,002)$.

Con respecto a la confianza en el control del aprendizaje, no se encontraron diferencias significativas entre el GE y GC. En cambio, si se encontraron diferencias estadísticamente significativas en el control de autoeficacia $(p=0,013)$. Los alumnos del GE creyeron que tendrían una buena nota en el práctico con una media de 4,29 frente a 3,56 en el GC $(p<0,001)$, entendieron en mayor medida los conceptos más complejos enseñados en la clase con una media de 4,36, en comparación con el GC que obtuvo una media de 3,92 ( $\mathrm{p}=0,011)$ y estuvieron más seguros de haber adquirido las habilidades necesarias para su práctica clínica con valores 4,14 frente a 3,68 del GC $(p=0,030)$.

\subsubsection{Escala de aprendizaje}

El análisis de las estrategias de aprendizaje se realizó en base a las respuestas de los alumnos a los 15 ítems de la escala del cuestionario CMEA, encontrándose respuestas similares en ambos grupos.

En cuanto a las estrategias de manejo de los recursos, el GE mostró niveles más altos con respecto a la regulación del esfuerzo, con una media de 4,31 frente a 3,51 en el GC ( $<<0,001)$ (Tabla 5). Concretamente, se encontraron diferencias estadísticamente significativas que reflejaron menor aburrimiento $(\mathrm{p}<0,001)$ y menos ganas de abandonar la clase por parte del GE $(\mathrm{p}<0,001)$.

Tabla 5. Puntuaciones sub-escala y escala de estrategias del aprendizaje del CMEA según grupo: resultados test Mann-Whitney (MW).

\begin{tabular}{rc}
\hline & p-valor \\
\hline PENSAMIENTO CRÍTICO & 0,462 \\
AUTORREGULACIÓN METACOGNITIVA & 0,389 \\
REGULACIÓN DEL ESFUERZO & $<\mathbf{0 , 0 0 1 * * *}$ \\
APRENDIZAJE PARALELO & 0,575 \\
BÚSQUEDA DE AYUDA & 0,134 \\
APRECIACIÓN GENERAL & 0,714 \\
\hline *p $<0,05 ; \quad * * \mathrm{p}<0,01 ;$ & $* * * \mathrm{p}<0,001$
\end{tabular}


En síntesis, los alumnos del GE, en comparación con el GC:

1. Tras la clase, se sintieron más capaces y con mayor confianza para afrontar las prácticas clínicas

2. Consideraron que el material les fue útil para el aprendizaje

3. Previeron una buena nota en la parte práctica

4. Entendieron los conceptos más complejos explicados en la clase

5. Estuvieron seguros de haber adquirido las habilidades necesarias para la práctica clínica

Del mismo modo, se mostraron menos de acuerdo, en comparación a los del GC, con:

6. Sensación de desgana y aburrimiento durante la clase

7. Ganas de abandonar la tarea antes de acabarla

\section{Conclusiones}

- El estudio demostró la mayor eficacia del método de aprendizaje combinado en términos de incremento de conocimientos. Los alumnos del GE aumentaron en 2,7 puntos la nota media del examen teórico, frente al GC, que sólo lo hicieron en 2,0 puntos. Las diferencias se situaron al límite de la significancia estadística. El resultado del examen práctico también fue más alto también en el GE, donde aspectos como la realización de la aspiración anestésica, la forma de coger el botador o la posición de la mano no operante fueron mejor adquiridos que en el GC.

- Los alumnos del GE se mostraron más satisfechos con aspectos como el contenido, organización, material, cobertura, y diseño de la clase que el GC. El 92,9\% del GE consideró que la metodología empleada les permitió entender la clase con facilidad, frente a solo el $64 \%$ del GC. En general, los alumnos del GE consideran que el aprendizaje combinado es más efectivo, motivador y promueve la auto-evaluación y el pensamiento crítico. La experiencia fue más instructiva y agradable para los alumnos del GE, que seguirán utilizando la web y la recomendarán en el $100 \%$ de los casos.

- Los alumnos del grupo GE presentaron una puntuación significativamente superior en la escala de motivaciones del CMEA, sobre todo, con respecto a la valoración de la tarea y control de la autoeficacia. Los alumnos de ambos grupos exhibieron una puntuación similar en la escala de estrategias del aprendizaje, encontrándose diferencias sólo en la regulación del esfuerzo. Así, el GE se vio más preparado para afrontar las prácticas clínicas, valoraron mejor el material y creyeron haber asimilado mejor los conceptos difíciles. Por el contrario, el GC experimentó más deseos de abandonar la sesión formativa.

(c)) EY-NC-ND 2017, Universitat Politècnica de València 


\section{Referencias}

ALY M, WILLEMS G, VAN DEN NOORTGATE W, ELEN J. (2011). "Effect of multimedia information sequencing on educational outcome in orthodontic training" en Eur J Orthodont, vol. 34 , issue. 4 , p. 458-65.

HU J, YU H, SHAO J, LI Z, WANG J, WANG Y. (2008). "Effects of dental 3D multimedia system on the performance of junior dental students in preclinical practice: a report from China" en Advances in Health Sciences Education, vol. 14, issue. 1, p. 123-133.

KAVADELLA A, TSIKLAKIS K, VOUGIOUKLAKIS G, LIONARAKIS A. (2011). "Evaluation of a blended learning course for teaching oral radiology to undergraduate dental students." en European Journal of Dental Education, vol. 16, issue. 1, p. 88-95.

KRUGER C, SKALEN M, HARJU-JEANTY D, HEYMANS R, ROSEN A, LUN B. (2016). "Implementation of a Web-Based Patient Simulation Program to Teach Dental Students in Oral Surgery” en J Dent Educ, vol. 80, issue. 2, p. 133-140.

LAVARÍAS RIBES, B., ODEH OLMEDA, V. Anestesia y cirugia bucal. <http://www. http://cirubuca.wixsite.com/ciruvestic> [Consulta: 30 de Marzo de 2014]

LINJAWI A, HAMDAN A, PERRYER D, WALMSLEY A, HILL K. (2009). 'Students' attitudes towards an online orthodontic learning resource” en Eur J Dent Educ, vol. 13, issue. 2; p. 87-92.

PEÑARROCHA DIAGO, M., SANCHÍS BIELSA, J.M., MARTÍNEZ GONZALEZ, J.M., (2010). "Técnicas anestésicas primarias" en Peñarrocha Diago, M., Martínez Gonzalez, J.M., Martí Bowen, E. Anestesia local en Odontología. Barcelona: Editorial Ars Medica.

MECKFESSEL S, STÜHMER C, BORMANN K, KUPKA T, BEHRENDS M, MATTHIES H. (2011). "Introduction of e-learning in dental radiology reveals significantly improved results in final examination" en J Craniomaxillofac Surg, vol. 39, issue 1, p. 40-8.

NEUHAUS K, SCHEGG R, KRASTL G, AMATO M, WEIGER R, WALTER C. "Integrated learning in dentistry: baseline data and first evaluation at the Dental School of Basel" en Eur J Dent Educ, vol. 12, issue 3, p. 163-69.

SANTOS G, LEITE A, FIGUEIREDO P. (2016). "Effectiveness of E-Learning in Oral Radiology Education: A Systematic Review” en J Dent Educ, vol. 80, issue. 9, p. 1126-39.

VARTHIS S, ANDERSON O. "Students' perceptions of a blended learning experience in dental education” en Eur J of Dent Educ, p. 1-7.

ZAMBRANO RAMÍREZ, J. "Factores predictores de la satisfacción de estudiantes de cursos virtuales" en Revista Iberoamericana de Educación a Distancia, vol. 19, issue. 2, p. 217-35. 